\title{
Novel Approach for Center Frequency and Bandwidth Tuning in Multimode Resonator Based Microstrip Dual- Mode Bandpass Filter
}

\author{
Shobha Hugar $^{1^{*}}$ and Vaishali B. Mungurwadi ${ }^{1}$ \\ ${ }^{I}$ Department of Electronics and Communication Engineering, Sapthagiri College of Engg, Bangalore, India \\ * Shobhahugar@yahoo.co.in
}

\begin{abstract}
In this paper, a novel approach for both center frequency and bandwidth tuning in a dual mode bandpass filter is demonstrated. The proposed filter is configured from a half wavelength multimode resonator structure. The Ultra-wide bandpass response of the multimode resonator is extracted using an inter-digital feed structure which provides good input/output coupling. By deploying stepped admittance structure perturbation element into the symmetrical plane of the multimode resonator, the dual-mode response is achieved with three upper stopband transmission zeros (TZs). The coupling between two degenerative mode frequencies is controlled by the admittance ratio (Y) of the stepped admittance structure. Changing admittance ratio $(\mathrm{Y})$ of stepped admittance structure, resulted in the change in even mode resonance frequency and location of three upper stopband transmission zeros while keeping odd mode frequency fixed. The proposed filter has a size of $14.0 \times 30.0 \mathrm{~mm}^{2}$.
\end{abstract}

Keywords: Multi-mode resonator, Ultra-wideband, Transmission Zeros.

\section{Introduction}

Modern wireless communication system needs compact, high frequency selective, wide stopband tunable bandpass filters. In literature many tunable filters have been reported using dual mode resonators [1]-[12].Tuning is achieved in 3 ways i) Fixed center frequency and tunable bandwidth ii) Fixed bandwidth and tunable center frequency iii) Both center frequency and bandwidth tuning. In literature, electronic tuning also has been reported where semiconductor and MEMs switches are used for center frequency and bandwidth tuning. By varying dimensions of perturbations in microstrip loop resonators even mode frequencies are controlled and thus bandwidth tuning has been reported in [5]-[7]. By varying size of $\mathrm{T}$ shaped DGS and inter-digital capacitance, center frequency of ultra wideband filter is controlled [2]. S-band microstrip triple mode bandpass [3] filter has been proposed with $\mathrm{T}$ shaped resonators, both upper and lower transmission zeros were controlled by tuning the length of the T resonator. Parallelcoupled stepped impedance resonators (SIRs) [4] have been proposed to design Bandpass filters with an optimal rejection bandwidth. Dual-mode bandpass filter using square loop resonator DGS [8] with square patch in corner has been proposed to excite odd and even modes. But filter suffers from poor Roll-off at lower passband edge. An open loop resonator [10] with inter-digital unit cells which provide capacitive loading effect has been used to configure dual mode filter. By varying gap between fingers and width of fingers odd and even mode resonant frequencies were observed. But filter suffers from poor frequency selectivity. Dual mode DGS resonator composed of folded slot line resonator (FSLR) and coplanar stepped impedance resonator (CSIR) has been proposed to develop filter. Each of the resonator controls one resonant frequency [12].
In this paper a novel approach for center frequency and bandwidth tuning in MMR based dual mode BPF is proposed. Using an inter-digital feed structure Ultra-wide bandpass response of multimode resonator is obtained. By deploying stepped admittance structure perturbation in to the symmetrical plane of multimode resonator, dual mode response is achieved with three upper stopband transmission zeros(TZs).Change in admittance ratio(Y) of stepped admittance structure, results in shift in even mode resonance frequency and location of three upper stopband transmission zeros.

\section{Modeling of UWB bandpass filter from MMR}

Basic structure of half wavelength MMR is depicted in Figure 1 which consists of low and high impedance sections $\mathrm{Z} 1$ and $\mathrm{Z} 2$ respectively and resonates at fundamental frequency $f_{0}=4.4 \mathrm{GHz}$ and at $2 f_{0}=10.14 \mathrm{GHz}$, and $3 f_{0}=14.6$ $\mathrm{GHz}$ as shown in Figure 2. By providing proper coupling at input and output through an inter-digital feed structure UWB filter is modeled as shown in Figure 3. Figure 4 shows response of UWB response. The proposed resonator is modeled using RT Duroid substrate with dielectric constant 10.8 and thickness $1.27 \mathrm{~mm}$ and tangential loss of 0.0023 .

Dual- mode bandpass filter is configured by deploying stepped admittance structure perturbation in to the symmetrical plane of proposed MMR as shown in Figure 5 which excites two degenerative odd and even mode resonant frequencies. As filter topology is symmetrical, odd and even mode resonant frequencies are analyzed based on the equivalent circuits shown in Figure 6 and 7, respectively.

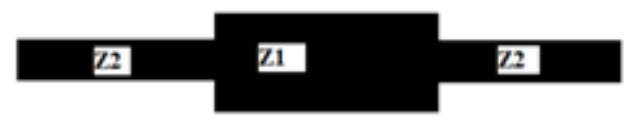

Fig.1.Structure of multimode resonator 


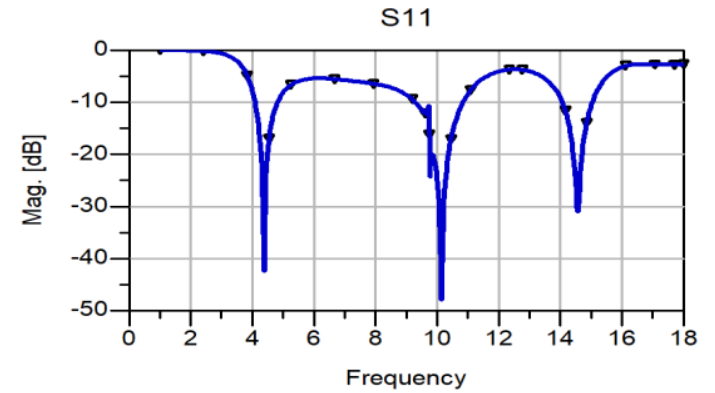

Fig.2. Resonance frequencies of MMR

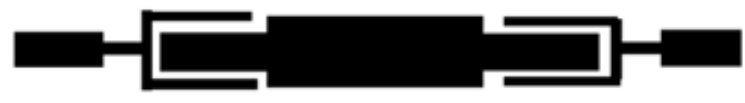

Fig.3. Proposed UWB Filter

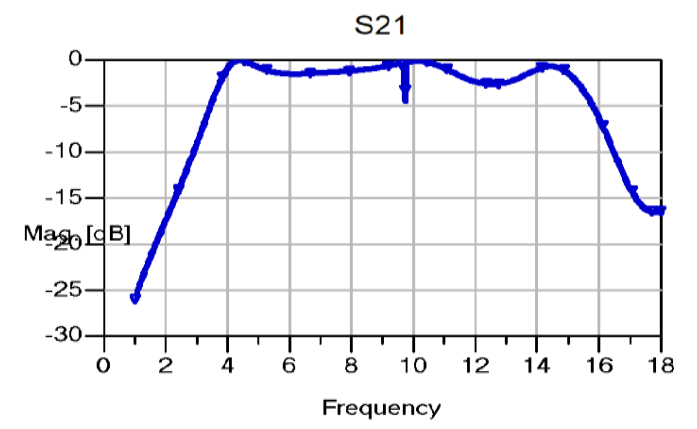

Fig.4. UWB response of MMR

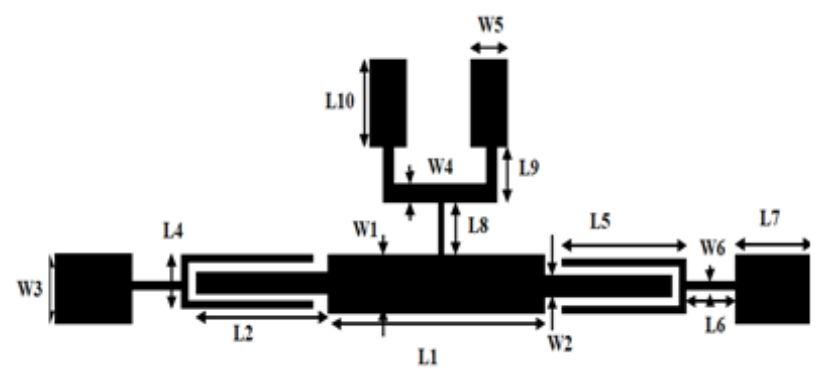

Fig.5. Proposed MMR based dual mode Bandpass filter

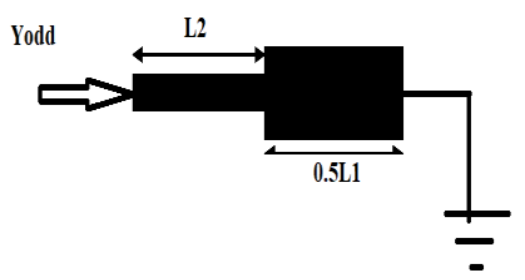

Fig.6. Equivalent circuit for odd mode resonance

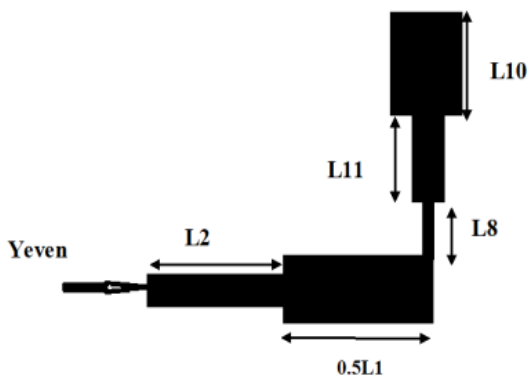

Fig.7. Equivalent circuit for even mode resonance
Mathematical model for Odd and even mode resonance frequencies are given by expressions (1) and (2) respectively.

$$
\begin{gathered}
f_{\text {odd }}=\frac{c}{\left(L_{2}+0.5 L_{1}\right) \sqrt{\varepsilon_{\text {eff }}}} \\
f_{\text {even }}=\frac{c}{\left(L_{2}+0.5 L_{1}+L_{8}+L_{11}+L_{10}\right) \sqrt{\varepsilon_{\text {eff }}}} \\
\varepsilon_{\text {eff }}=\frac{\varepsilon_{r}+1}{2}+\frac{\varepsilon_{r}-1}{2}\left[\sqrt{1+\frac{12 h}{t}}\right]^{-1}+0.04\left(1-\frac{w}{h}\right)^{2}
\end{gathered}
$$

where, $\mathrm{c}$ is velocity of light, $\varepsilon_{\text {eff }}$ effective dielectric constant. For an admittance ratio (Y) of 0.8 , the response of filter is shown in Figure 8. Odd and even mode frequencies are found at $4.55 \mathrm{GHz}$ and $6.0 \mathrm{GHz}$, respectively with three upper stopband transmission zeros at $8.88 \mathrm{GHz}, 9.75 \mathrm{GHz}$ and $10.86 \mathrm{GHz}$. From simulation results, it is observed that the designed filter has input reflection coefficient $\left(\mathrm{S}_{11}\right)>$ $10 \mathrm{~dB}$ and transmission loss $\left(\mathrm{S}_{21}\right)-0.08 \mathrm{~dB}$ in pass band and bandwidth of $3.4 \mathrm{GHz}$. The dimensions of proposed filter are as follows.

$\mathrm{L}_{1}=5.7 \mathrm{~mm}, \mathrm{~W}_{1}=0.8 \mathrm{~mm}, \mathrm{~L}_{2}=2.7 \mathrm{~mm}, \mathrm{~W}_{2}=0.5 \mathrm{~mm}, \mathrm{~W}_{3}=1.1$ $\mathrm{mm}, \mathrm{W}_{4}=0.33 \mathrm{~mm}, \mathrm{~L}_{4}=0.7 \mathrm{~mm}, \mathrm{~L}_{5}=2.7 \mathrm{~mm}, \mathrm{~W}_{5}=(0.6,0.7$, $0.9) \mathrm{mm}, \mathrm{W}_{6}=0.1 \mathrm{~mm}, \mathrm{~L}_{6}=0.2 \mathrm{~mm}, \mathrm{~L}_{7}=2 \mathrm{~mm}, \mathrm{~L}_{8}=0.2 \mathrm{~mm}$, $\mathrm{L}_{9}=0.3 \mathrm{~mm}$ and $\mathrm{L}_{10}=1.4 \mathrm{~mm}$.

\section{Tuning Center frequency and Bandwidth}

Both center frequency and bandwidth of the proposed filter are tuned by varying admittance ratio(Y) of stepped admittance perturbation as $0.7,0.77$ and 0.8 . From simulation results shown in Figure 9. it is observed that, even mode resonance frequency changes with change in admittance ratio where as the odd mode resonance frequency remains fixed. In Figure 10 shows shift in position of upper stop band transmission zeros with changing admittance ratio (Y).

\section{Result and discussion}

To tune both center frequency and bandwidth, admittance ratio $\mathrm{Y}$ is varied as $0.7,0.77$ and 0.8 . For $\mathrm{Y}=0.7,0.77$, and 0.8 , even mode resonance frequencies are found at 5.75 $\mathrm{GHz}, 6.0 \mathrm{GHz}$, and $6.1 \mathrm{GHz}$ respectively. Whereas for odd

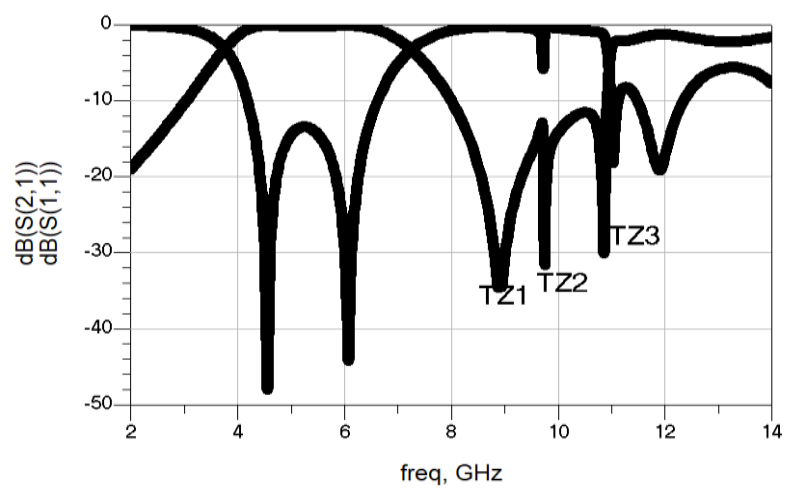

Fig.8. Response of proposed filter for $\mathrm{Y}=0.8$ 


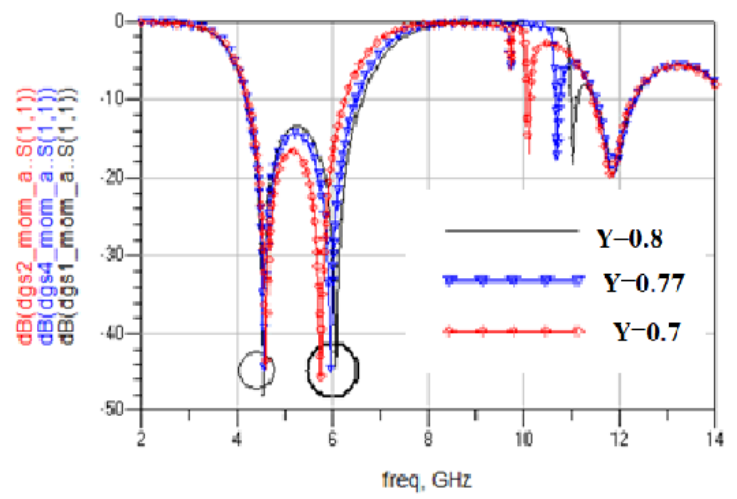

Fig.9. Shift in even mode resonant frequency.

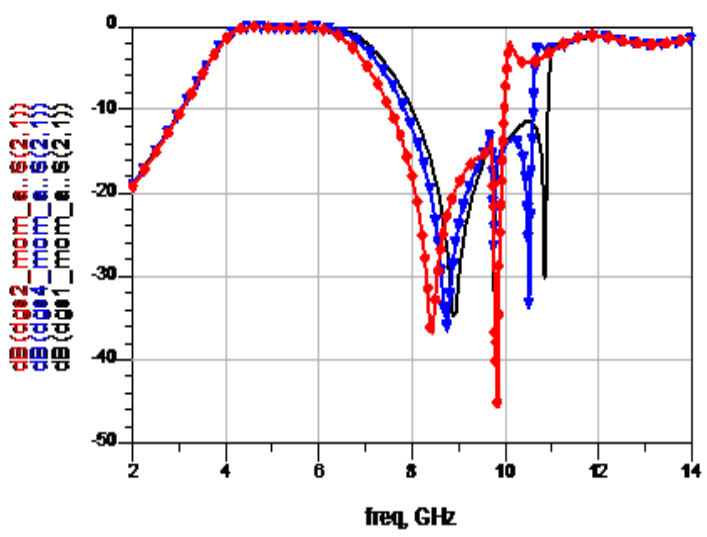

Fig.10. Shift in position of upper stop band transmission zeros in $\mathrm{S}_{21}$ response.

Tablel 1: Measured values of center frequency and bandwidth for various values of admittance ratio (Y)

\begin{tabular}{|l|l|l|l|}
\hline $\begin{array}{l}\text { Admittance } \\
\text { Ratio (Y) }\end{array}$ & $\begin{array}{l}\text { Center } \\
\text { frequency in } \\
\text { GHz }\end{array}$ & $\begin{array}{l}\text { Bandwidth } \\
\text { in GHz }\end{array}$ & $\begin{array}{l}\text { Location of Upper } \\
\text { stopband transmission } \\
\text { zeros at }\end{array}$ \\
\hline 0.7 & 5.157 & 3 & $\begin{array}{l}8.44 \mathrm{GHz} \text { and } \\
9.8 \mathrm{GHz} .\end{array}$ \\
\hline 0.77 & 5.3 & 3.2 & $\begin{array}{l}8.750 \mathrm{GHz}, 9.75 \mathrm{GHz} \\
\text { and } 10.50 \mathrm{GHz}\end{array}$ \\
\hline 0.8 & 5.4 & 3.4 & $\begin{array}{l}8.750 \mathrm{GHz}, 9.75 \mathrm{GHz} \\
\text { and } 10.50 \mathrm{GHz}\end{array}$ \\
\hline
\end{tabular}

mode frequency is at $4.6 \mathrm{GHz}$ for all values of $\mathrm{Y}$. For $\mathrm{Y}=0.7$ two upper stop band transmission zeros are reported at 8.44 $\mathrm{GHz}$ and $9.8 \mathrm{GHz}$. For $\mathrm{Y}=0.77$, three upper stop band transmission zeros are reported at $8.750 \mathrm{GHz}, 9.75 \mathrm{GHz}$, and $10.50 \mathrm{GHz}$, respectively. For $\mathrm{Y}=0.8$, three upper stop band transmission zeros are reported at $8.75 \mathrm{GHz}, 9.75 \mathrm{GHz}$ and $10.7 \mathrm{GHz}$, respectively. The input reflection coefficient $\left(\mathrm{S}_{11}\right)>-10 \mathrm{~dB}$ and transmission loss $\left(\mathrm{S}_{21}\right)$ of $-0.07 \mathrm{~dB}$ are reported for $\mathrm{Y}=0.7,0.77$, and 0.8 .

\section{Conclusion}

In proposed work, dual-mode bandpass filter is realized from multimode resonator. Dual mode response is achieved by integrating an stepped admittance perturbation in to the MMR. By changing admittance ratio $\mathrm{Y}$ of stepped admittance perturbation, center frequency and Bandwidth are tuned and three upper stopband transmission zeros are created. The location of all upper stopband transmission zeros are controlled by admittance ratio.

\section{References}

[1] Hongshu Lu, Tao Xie, Jingjian Huang, Naichang Yuan, Qian $\mathrm{Li}$, Design of compact dual-mode bandpass filter based on a new fractal resonator, Proc. IEEE International Symposium on Microwave, Antenna, Propagation, and EMC Technologies (MAPE) 2017.

[2] Arjun Kumar and M.V. Kartikeyan, Design studies of ultra wideband microstrip bandpass filter with $\mathrm{T}$-shaped defected ground structure controlled by inter-digital capacitance, IEEE conference on Applied Electromagnetics (AEMC) 2011.

[3] Ran Liu, Li Zhi Li, and Wen Shi, Compact microstrip triplemode filter based on stepped-impedance resonators, IEEE

International Conference on Electronic Packaging Technology (ICEPT) 2014.

[4] J.-T. Kuo and E. Shih, Microstrip stepped impedance resonator bandpass filter with an extended optimal rejection bandwidth, IEEE Transactions on Microwave Theory and Techniques 2003.

[5] T. Y. Xiang, T. Lei, and M. Peng, Miniature dual-mode bandpass filter based on meander loop resonator with sourceload coupling, Asia-Pacific Microwave Conference (APMC) 2015.

[6] Sugchai Tantiviwat, Mohammad Shahrazel Razalli, and Siti Zuraidah Ibrahim, Miniature microstrip bandpass filters based on quadruple-mode resonators with less via IEEE MTT-S International Conference on Microwaves for Intelligent Mobility (ICMIM) 2017.

[7] C. Hua, C. Chen, C. Miao, and W. Wu, Microstrip bandpass filters using dual-mode resonators with internal coupled lines, Progress In Electromagnetics Research C, vol. 21, pp. 99111, 2011.

[8] Bian Wu, Xin Lai, Jia Chen, Xue-Feng Li, and Chang-Hong Liang, Dual-mode filter with asymmetric response using square loop resonator DGS, IEEE Proceedings, ICMMT2010, 2010.

[9] K.G.Avinash and I.Srinivasa Rao, Design of microstrip meandered dual mode wideband bandpass filter using quarter wavelength stub, IEEE International Conference on Advances in Computing and Communications (ICACC) 2015.

[10] C. Karpuz, P. Ö. Özdemir, and G. B. Firat, Design of fourth order dual-mode microstrip filter by using interdigital capacitive loading element with high selectivity, IEEE European Microwave Conference (EuMC) 2016.

[11] D. Sen and D. Packiaraj, Design of drop in type band pass filter with extended stop, Proc. IEEE Asia-Pacific Microwave Conference (APMC), 2016.

[12] B. Peng, S. Li, B. Zhang, and S. Wang, Compact multimode bandpass filters with wide upper stopband using dual-mode DGS resonators Proc. IEEE Asia-Pacific Microwave Conference (APMC), 2014.

\section{Biography of the authors}

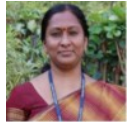

Shobha Hugar: She did B.E. in Elecetronics and Communication from Karnataka University Dharwadr and M.Tech. in VLSI Design \&Embedded System from Visvesvaraya Technological University in 2000 and 2009 respectively. Presently she is working as Assistant Professor at Department Of Electronics \& Communication Engineering, Sapthagiri College of Engineering, Banglore, Karnataka. Her reaserch area includes UWB filters and microstrip antennas.

Vaishali B. Mungurwadi:Not available 